\title{
基于动态化学的自愈性水凝胶及其在生物医用材料中的应用研究展望
}

\author{
张亚玲杨斌许亮金金张小勇陶磊*危岩* \\ (清华大学化学系 北京 100084)
}

\begin{abstract}
摘要 自愈性材料具有自我修复损伤的特点, 能够增加使用材料的安全性, 延长材料寿命, 是一种具有损伤管理性能 的智能新材料. 基于动态化学的自愈性水凝胶是近来备受关注的一种自愈性材料, 由具有动态特性的交联网络构建形 成. 交联作用为动态化学键, 即非共价键, 如弱相互作用的氢键、分子间作用力(范德华力)、配位作用、亲疏水作用等, 或可逆共价键, 如温和条件下可逆的亚胺键、双硫键、酰腙键等. 这种材料具有本征性的自愈性, 一方面可应对外界破 坏造成的损伤, 进行自我修复. 另一方面动态化学键对多种环境刺激具有响应性, 能自我调节以适应环境变化, 为将 自愈性水凝胶开发为自适性多功能智能新材料奠定了基础. 水凝胶具有优越的生物相容性以及和生物组织的相似性, 在生物医用材料中如药物控制释放、组织工程修复、生物仿生等领域发挥着越来越大的作用, 而开发具有自愈性的多 功能智能水凝胶, 将进一步拓展其应用. 综述了近来基于动态化学的自愈性水凝胶的制备及其在生物医用材料领域中 的应用研究.
\end{abstract}

关键词 自愈性; 自适性; 水凝胶; 动态化学; 智能软物质

\section{Self-healing Hydrogels Based on Dynamic Chemistry and Their Bio- medical Applications}

\author{
Zhang, Yaling Yang, Bin Xu, Liangxin Zhang, Xiaoyong Tao, Lei* Wei, Yen* \\ (Department of Chemistry, Tsinghua University, Beijing, 100084)
}

\begin{abstract}
Self-healing materials are able to repair themselves automatically after external normal damages. This special property of self-healing materials could enhance the service life and ensure more safety while using such materials especially mechanically. Therefore, it has become a new emerging smart material which has the specialty to manage damages instead of traditional materials to avoid it. Self-healing hydrogels based on constitutional dynamic chemistry have received a lot of attentions recently with their intrinsic dynamic structures namely cross-linked polymer networks by dynamic bonds. Dynamic chemistry bonds contain both non-covalent bonds and reversible covalent bonds. Non-covalent bonds (weak effects) include hydrogen bonds, van der Waals forces, coordinative bonds, hydrophobic effects, etc. Reversible covalent bonds usually include those chemistry bonds that could be reversible in mild conditions as imine bonds, disulfide bonds, acylhydrazone bonds and so on. Those hydrogels with dynamic cross-linked networks could not only manage external damages and repair themselves as self-healing materials but also gain multi-responsive properties to environmental stimuli. The latter specialty comes from the adptive property of dynamic bonds to react to changes of reaction environments like $\mathrm{pH}$, temperature and chemical reactants, thus building the foundation to develop self-healing hydrogels further into a multi-functional self-adaptive smart soft matter, which is of great significance for research to enrich multi-functional materials. Besides, hydrogel as a soft matter have long been vastly used in biomedical applications due to their superior biocompatibility and resemblance to biological tissues as mainly components (usually more than 70\%) of hydrogel are water. They are playing a more and more important role in biomedical applications such as drug delivery systems, cell culture, tissue engineering and manmade biomimetic materials. Developing multi-functional smart soft matter with self-healing property as self-healing hydrogels would be quite helpful to this emerging field with unexpected more biomedical materials. This paper reviews recent works about self-healing hydrogels based on dynamic chemistry and their future biomedical applications. Systems based on multiple-hydrogen bonds, coordination effects, hydrophobic effects, acylhydrazone bonds and imine bonds are specifically discussed.
\end{abstract}

Keywords self-healing; self-adaptive; hydrogel; dynamic chemistry; smart soft matter

\section{1 引言}

众所周知, 生物组织受损时, 会有极为复杂而高效 的自修复过程发生, 模仿此过程的新型材料即为备受关
注的自愈性材料. 自愈性材料的开发可以提高相关材料 使用的安全性，延长材料的使用寿命等，在近 10 年来的 研究中受到了越来越多的关注 ${ }^{[1 \sim 4]}$.

由于高分子材料具有可调节的化学结构和机械性

\footnotetext{
*E-mail: leitao@mail.tsinghua.edu.cn; weiyen@tsinghua.edu.cn; Tel.: 010-62792604; Fax: 010-62794694 Received January 28, 2013; published March 5, 2013.

Project supported by the National Natural Science Foundation of China (Nos. 21104039, 21134004) and the National 973 Project (No. 2011CB935700). 项目受国家自然科学基金(Nos. 21104039, 21134004)及国家 973 项目(No. 2011CB935700)资助.
} 
能, 在一定尺度上通过对聚合过程的控制, 可以较容易 地实现对材料性能的控制, 自愈性高分子材料的研发得 到了迅速发展. 早期的自愈性高分子材料主要集中于微 胶囊、微管破裂引发再聚合实现修复损伤 ${ }^{[2]}$, 目前的研 究更多集中在通过对材料本身的结构设计实现对材料 损伤的自修复. 早期材料的自愈性可以算是修补过程, 由于再聚合时需要引发相应的单体实现损伤部位的修 补, 因此这种自愈性通常只能实现一两次, 无法重复修 复损伤. 目前的研究则倾向于利用材料本身物理化学结 构的特点, 在一定刺激因子和外界能量输入下, 启动自 身结构变化达到自愈的效果. 这种自愈材料有赖于结构 设计上的动态特征, 即目前较受认可的流动相机理 ${ }^{[3,4]}$, 材料产生流动相以分散外界能量造成的冲击, 并使材料 在维持其骨架的情况下重新分配能量和物质达到新的 平衡态. 如何使得材料结构具有一定无序可变的动态性 质是制备自愈合材料的关键, 即如何使得材料具有一定 比例的流动相, 并且可以在特定条件下动起来. 这方面 的研究者在结构设计与调控上做了许多新颖而且成果 显著的尝试. 例如, 在最近的工作中, Wojtecki 等 ${ }^{[5]}$ 利用 动态组合化学, 在高分子凝胶网络中引入动态非共价键 以及超分子弱相互作用, 使得材料具有本征性的自愈特 性. 又如 Chen 等 ${ }^{[6]}$ 在高分子弹性体中设计同时有软硬 多相的一个结构, 包括比较硬而强度大的固定相, 在受 到作用力时可以保持材料的骨架结构, 而其中比较软的 流动相则产生一定的结构流动, 或者宏观上称为形变来 缓解材料本身的应力, 实现材料修复.

高分子凝胶中的水凝胶, 作为一种越来越受关注的 软物质, 通常指在水溶液中溶胀的高分子相互交联而形 成的三维网络结构. 由于其组成大部分为水, 水凝胶与 生物组织极为相似，水凝胶含有的水自然充当了相应的 流动相, 在实现材料的自愈性方面有自己独特的优势 ${ }^{[3]}$. 此外, 水凝胶可控的交联结构和密度使其力学性质有较 广的可调节范围. 从较弱的物理交联到较强的共价化学 键交联结构, 水凝胶本身的力学强度则表现出介于溶液 态和高交联度的凝胶态二者之间的性质，从而影响了流 动相比例和材料的自愈性.

当水凝胶接近溶液态时, 体系的相转变能垒较低, 外界给予微弱刺激即可产生流动相, 从而达到自愈的目 的，中国古诗中 “抽刀断水水更流” 的诗句即表明水溶 液这种极稀溶液具有极低的的能垒, 处于一种自由流动 的状态. 对于高分子溶液来说, 由于高分子长链间存在 相互缠结, 溶液粘度较大, 流动缓慢, 能垒变高, 表现 出粘弹性的特征. 高分子溶液经交联达到凝胶态时, 随 着键和强度及交联度的提高, 能垒进一步变高, 需要外 界环境给予较高的能量如热、光刺激, 或者一些化学刺 激如 $\mathrm{pH}$ 值的改变等, 才能使得材料结构中产生流动相, 实现宏观形态的改变达到自愈合. 而对于结构有序度与 稳定性更高的固体材料, 如金属、陶瓷等, 则需要外界
施加诸如高温等更高的能量才能产生流动相 ${ }^{[1,7]}$. 可以 说，材料的力学强度来自于结构的稳定性，而其实现自 愈则需要本身产生流动相，二者是相互矛盾与相互协同 的关系. 通常单一有序结构的材料, 其力学强度较大, 宏观表现为材料较硬, 产生流动相较难, 实现自愈也较 难. 但是, 即使对于比较软而脆弱的材料如水凝胶, 如 果没有巧妙的结构设计，也并不能获得良好的自愈性， 如普通的明胶、果胶类水凝胶，结构内缺少分子链间相 互作用，交联结构不具有动态特性，体系中仍然缺乏流 动相，故而难以实现自愈.

将自愈性这一特点引入在生物医用材料中得到广 泛应用的水凝胶，可以进一步增加水凝胶的功能，拓展 其在生物领域的应用, 并且具有重要的仿生意义. 如自 愈性水凝胶很好地满足了可注射水凝胶的要求 ${ }^{[8]}$, 在注 射过程中被破坏了的水凝胶可在生物体内实现自愈，避 免了手术植入, 具有较长的使用寿命, 并且可装载多种 生物活性物质. 此外, 通过结构设计使自愈性水凝胶具 备多种生物刺激响应性，可以开发出多功能的智能医用 水凝胶. 生物医用水凝胶需要在生理条件下使用, 基于 这一特点，生物医用自愈性水凝胶倾向于本征自愈性， 即材料本身不借助于外界刺激或能量输入，自适性的实 现动态调节. 目前研究的自愈性水凝胶有相当一部分是 在外缘刺激下，如热、光等促进作用下实现修复过程. 外界刺激在生物体内的使用有极大的限制, 如微小的温 度变化即有可能对生物组织产生不可逆危害，限制了热 促自愈性水凝胶的使用. 又例如光促修复的水凝胶，由 于光的穿透性有限而难以高效实现材料在体内修复. 因 此, 将自愈性水凝胶应用于生物医药领域, 较理想的情 况是采用无需借助外界能量输入的自发愈合体系，即基 于动态建构化学, 设计具有动态交联网络结构的自愈性 水凝胶. 超分子化学之父 $\mathrm{Lehn}^{[9]}$ 提出动态建构化学这一 概念并做了深入研究, 这一概念包括了动态共价化学和 超分子化学，其中很多化学键都具有条件可逆平衡和对 环境响应的特性，因此得到了广泛的关注. 动态建构化 学在水凝胶制备中的应用促进了自愈性水凝胶的发展， 研究者采取了基于不同动态化学键的体系，包括基于超 分子化学中弱相互作用力的物理型自愈性水凝胶和基 于动态共价化学的化学型自愈性水凝胶. 下面就将对最 近研究中的由不同动态化学键构筑的自愈性水凝胶其 所采用的材料和自愈机理做研究综述, 并对其在生物医 用材料领域的应用做一些展望.

\section{2 基于动态化学的自愈性水凝胶}

\section{1 多重氢键作用}

多重氢键作用在自愈性高分子弹性体中得到了广 泛的应用 ${ }^{[6,10,11]}$, 在水凝胶体系中由于氢键作用较弱, 并且体系中大量存在的水会影响氢键，使得以氢键作主 体交联的材料强度较弱而应用受限. 在新近的研究中, 
Cui 等 ${ }^{[12]}$ 用可由氢键形成二聚体的 2-嫝基-4-嘧啶酮 (UPy)与水溶性高分子单体如 2-二甲氨基丙烯酸乙酯 (DMAEMA)等共聚, 得到侧链带有 UPy 基团的高分子. 高分子链上的UPy 基团相互间存在多重氢键作用, 当体 系的 $\mathrm{pH}$ 值由酸性调为碱性 $(\mathrm{pH}>8)$ 时, 氢键作用加强, 高分子交联形成三维网络结构的水凝胶. 通过在石英微 天平上(QCM)修饰 UPy 基团研究了共聚物间氢键的相 互结合能. 进一步用铌子将水凝胶进行撕扯破坏, 观察 损伤后自愈的过程. 在较低温度下, 自愈可以快速实现, 观察到该水凝胶体系具有较强的流动性, 可用于进一步 的可注射性水凝胶的应用. 温度高于聚 DMAEMA 链的 临界转变温度 $\left(\mathrm{LCST} \approx 50{ }^{\circ} \mathrm{C}\right)$ 时, 高分子链舒展, 链间 距离变大, UPy 基团间的氢键作用变弱, 使得水凝胶无 法自愈而具备了温度调控自愈性的特点(图 1) ${ }^{[12]}$. 此外, 研究者通过与不同单体共聚将 UPy 基团引入不同的高 分子链, 观察UPy 基才能否在一个比较宽的范围内赋予 这些高分子水凝胶体系以自愈性, 以获得一种以 UPy 基 才修饰的高分子制备多重氢键自愈性材料的普适方法.

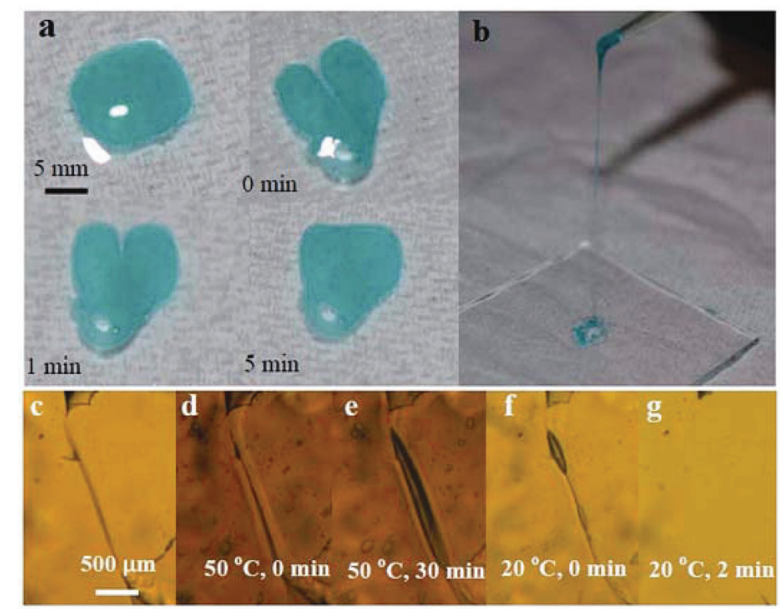

图 1 DMAEMA-SCMHBMA 水凝胶在 $\mathrm{pH}=8$ 条件下自愈性(a, c, d, $e, f, g)$ 和拉伸性(b) 表现 ${ }^{[12]}$

Figure 1 Demonstration of the self-healing (a, c, d, e, f, g) and the stretching (b) properties of the DMAEMA-SCMHBMA hydrogel at $\mathrm{pH}=$ $8^{[12]}$

Gel in (a) and (b) was coloured with methyl blue for better imaging. Optical microscopy images $(\mathrm{c} \sim \mathrm{g})$ were obtained from a hydrogel film with an incision (c) after annealing at $50{ }^{\circ} \mathrm{C}(\mathrm{d}, \mathrm{e})$ and subsequent cooling to $20{ }^{\circ} \mathrm{C}$ (f, g)

此外, 氢键常用以辅助自愈, 并配合其他交联作用 用以制备具有一定力学强度的、可自愈的水凝胶. 如借助 无机粘土原位聚合的高分子自愈性水凝胶 ${ }^{[13]}$, 复合作用 的网络交联结构使得材料力学性能较好, 而粘土片层与 高分子链间的氢键作用以及高分子链间的相互缠结作用 等弱相互作用使得水凝胶网络结构具有动态特性. 实验 结果表明, 不论水凝胶体系的断面新鲜与否, 稍微提高 温度 $\left(\approx 50{ }^{\circ} \mathrm{C}\right)$ 即在宏观上表现出良好的自愈性.

Phadke 等 ${ }^{[14]}$ 将疏水性的氢键引入侧链, 主链发生 共价交联得到力学强度较好的水凝胶, 而具有一定长度
的侧链可灵活伸展，侧链相互间的氢键作用则赋予体系 受损之后的自愈性. 由于氢键的数目较少并且采用的羧 基间氢键结合较弱，该水凝胶的自愈性在酸性条件下可 很快实现，而在碱性条件下该水凝胶则不具备自愈性. 研究者还同时探索了该自愈性水凝胶在生物医用材料 方面可能的应用, 主要包括修复涂层和组织粘合剂如胃 粘膜修复等, 为自愈性材料的具体应用打开了思路.

\section{2 金属配位相互作用}

金属配位相互作用由于金属离子与其配体的可选 范围广、响应快、可调控、理论基础丰富等特性被广泛 应用于自愈性材料中 ${ }^{[15 ~ 17]}$. 多巴胺, 作为一种生物配 体, 由于其独特的生物粘附性在近来的研究中广受关 注，而多巴胺的儿茶酚基团与金属离子存在着可以通过 酸碱变化调控的配位作用, 这一作用也被应用于动态自 愈性水凝胶的研究中 ${ }^{[18]}$. Holten-Andersen 等 ${ }^{[19]}$ 利用多巴 胺与三价铁离子 $\left(\mathrm{Fe}^{3+}\right)$ 在不同 $\mathrm{pH}$ 值环境下配位强弱的 不同，制备了 $\mathrm{pH}$ 诱导的金属配位型自愈性水凝胶. 随 着 $\mathrm{pH}$ 值增大，多巴胺与 $\mathrm{Fe}^{3+}$ 发生一配位到稳定三配位 情况的转变, 引起凝胶形态的变化.

四臂聚乙二醇通过修饰后端基带上多巴胺基团, 多 巴胺基团与金属离子配位后充当体系交联点制备得到水 凝胶. 该水凝胶的流变行为在酸性情况下 $(\mathrm{pH} \approx 5)$ 类似流 体, 当环境 $\mathrm{pH}$ 值增大到碱性时 $(\mathrm{pH} \approx 8)$, 体系粘度变大, 碱性进一步增大时 $(\mathrm{pH} \approx 12)$, 得到具有类似共价交联弹 性模量的水凝胶，同时伴随着凝胶颜色由深绿色向深红 色的逐渐转变. 紫外吸收光谱监测到了体系中金属离子 由一配位到三配位的转变. 流变仪监控施加破坏后的水 凝胶，证实其弹性模量可在短时间内恢复原本数值，而 作为对比的共价交联型水凝胶无法恢复(图 2), 体现了该 水凝胶的自愈性. 该工作 ${ }^{[19]}$ 提供了一种以多巴胺基团修 饰高分子网络制备动态结构材料的方法, 利用儿茶酚基 才与金属离子在不同 $\mathrm{pH}$ 值条件下发生强度不同的配位 而得到 $\mathrm{pH}$ 诱导型水凝胶. 该水凝胶的交联位点是由金属 配位作用得到的，较共价作用弱而又比氢键稍强，并同 时具有动态特性，使得制备的水凝胶材料具有自愈性的 同时，具备类似共价交联的模量，并且对环境的 $\mathrm{pH}$ 值具 有自适性变化. 多巴胺与其他多种金属离子也有类似的 配位作用，并且多巴胺本身具有的生物粘附等贻贝仿生 的特性，使得该体系还有广阔的开发空间.

\section{3 亲疏水相互作用}

Tuncaboylu 等 ${ }^{[20]}$ 通过胶束中引发自由基聚合合成 了亲疏水相互嵌段的高分子，制备了同时具有良好拉伸 性能和自愈性的强韧水凝胶. 在十二烷基硫酸钠(SDS) 胶束中加入盐如 $\mathrm{NaCl}$, 使得长链的疏水性单体如 $\mathrm{C}_{18}$ 和 $\mathrm{C}_{22}$ 等能够更多地增溶到胶束中, 并在接下来的聚合当 中进一步与亲水性单体如丙烯酰胺发生共聚. 疏水链段 在水溶液中发生聚集形成交联点, 得到非共价交联的物 


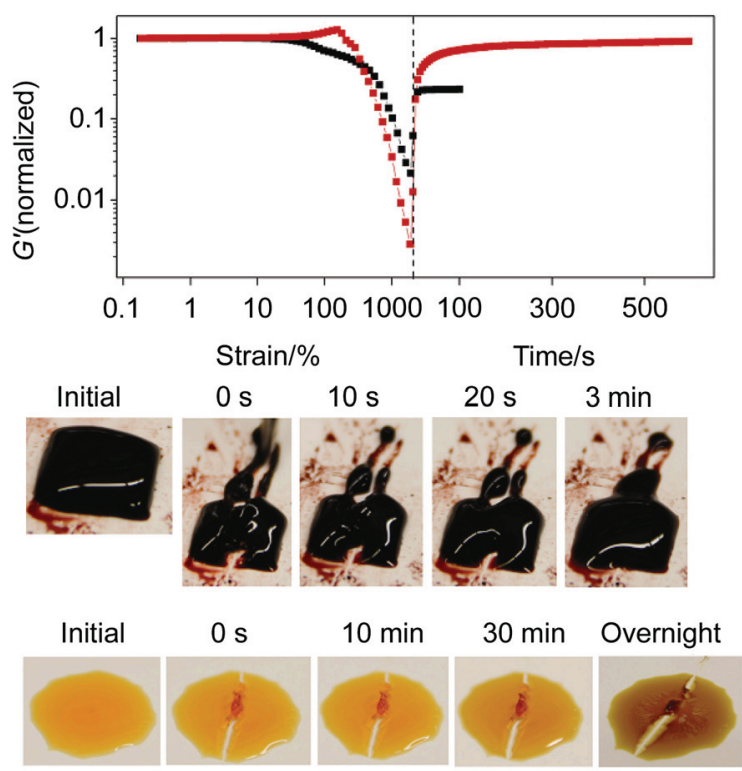

图 $2 \mathrm{Fe}^{3+}$ 与多巴胺三配位凝胶(红色曲线与上排图片)和共价交联凝 胶(黑色曲线与下排图片)经由剪切或铝子撕拉破坏后的力学性能恢复 过程 ${ }^{[19]}$

Figure 2 Recovery of stiffness and cohesiveness after failure induced by shear strain or simple tearing with the tip of a set of tweezers of tris-catechol- $-\mathrm{Fe}^{3+}$ gels (red curve and top row of images) and covalent gels (black curve and bottom row ofimages) ${ }^{[19]}$

Gels were shear failed under increasing oscillatory strain immediately followed by recovery under $1 \%$ strain (switch-over indicated by dashed line) while monitoring the storage modulus $\left(G^{\prime}\right.$ is normalized to values from linear regime, $<60 \%$ strain, to allow easier comparison)

理型水凝胶. 形成的水凝胶含有大量的疏水链段, 不会 在大量水溶液中发生崩解. 但是当在大量的胶束溶液中 时, 疏水相互作用在胶束中会变弱, 进而导致交联点崩 解而造成凝胶溶胶的转变. 由于该水凝胶交联点主要是 物理性的疏水作用，具有动态特性，当受到外力作用时 能够在受到破坏后又迅速形成. 因此该水凝胶有强㓞的 拉伸性能 $(\approx 3600 \%)$, 当受到外力发生断裂后, 断面相 互接触, 即可实现自愈, 自愈后的材料其拉伸性能没有 受到影响(图 3). 同时, 材料中亲疏水性单体的比例可 调, 以及在体系中加入的盐的浓度可调, 使得材料的自 愈性与拉伸性在一定范围内可调. 他们又研究了胶束的 存在对材料力学性能和自愈性的影响 ${ }^{[2]}$, 发现只有在 胶束存在的情况下, 材料因为具有灵活可动的交联位点 而具有很好的拉伸性并且可自愈, 当体系中的胶束通过 浸泡在大量水中一段时间后被除掉的时候, 链段之间的 疏水作用变强, 水凝胶的网络结构变得相对稳固而更加 类似共价交联的情况, 因此拉伸性能和自愈性都受到影 响. 我们注意到如此强韧而且具备自愈性的材料类似人 体肌肉组织, 但是生物相容性的问题还有待体系的进一 步调整. 同时在生物体系中如蛋白纤维等也广泛存在亲 疏水相互作用, 如何将其利用起来制备相关的生物医用 材料, 胶束增强的自愈性水凝胶提供了一种思路.

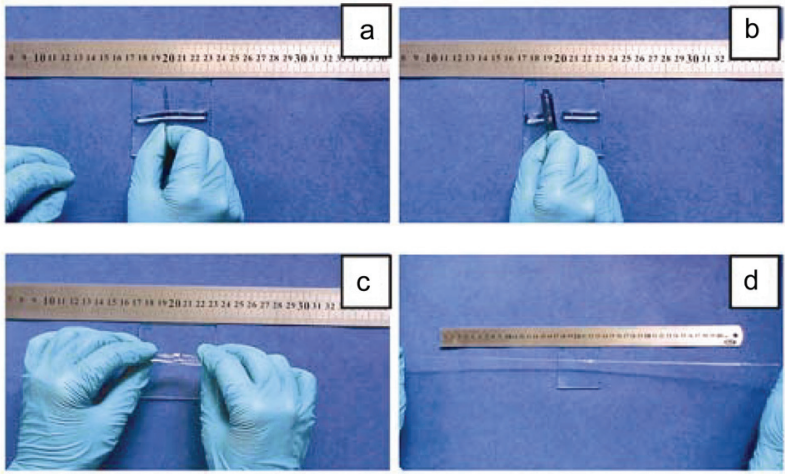

图 3 在 10\%浓度下形成的凝胶照片(该凝胶被切成两段后, 断面挤压 接触 $10 \mathrm{~min}$ 后愈合成一段 $)^{[21]}$

Figure 3 Photographs of a gel sample formed at $C_{0}=10 \%$ (After cutting into two pieces and pressing the fractured surfaces together for 10 min, they merge into a single piece) ${ }^{[21]}$

类似的借助较弱的超分子作用构筑动态三维网络 结构制备自愈性材料的体系还有主客体相互作用 ${ }^{[22 ~ 24] 、}$ $\pi-\pi$ 作用 ${ }^{[25,26]}$ 等. 前者一般需要使用较为昂贵的配体, 而后者体系中存在的 $\pi$ 基团一般水溶性较差，在水凝胶 中的报道较少.

\section{4 酰腙键}

酰腙键在动态非共价键中属于结合较稳定的一类. Deng 等 ${ }^{[27]}$ 利用两端酰阱封端的聚乙二醇(PEG)与三端修 饰了苯甲醛基的小分子交联剂，在酸催化下形成了具有 一定力学强度、并且具有酸碱响应特性的凝胶. 该凝胶利 用了酰腙键的动态平衡和小分子交联剂在界面间的扩散, 在室温下断面相互接触保持一段时间即可实现自愈.

此后, Deng 等 ${ }^{[28]}$ 又在此基础上引进了另一动态多响 应的双硫键，形成了同时具有酸碱和氧化还原双重响应 性的水凝胶. 他们合成了三端修饰苯甲醛基的三臂聚乙 二醇，与中间为双硫键连接的两端酰阴的小分子交联剂 在室温下于缓冲液中形成水凝胶, 成胶时间随缓冲液的 $\mathrm{pH}$ 值从 $2 \mathrm{~min}$ 到 $90 \mathrm{~min}$ 不等. 形成的水凝胶在水溶液中 长时间浸泡不会产生崩解，证实了酰腙键构成了相对稳 定的网络结构. 流变测试表征了该水凝胶的力学性能和 实现自愈的基础，具体分析了其流变行为与结构特点及 自愈性的关系. 在不同 $\mathrm{pH}$ 值条件下, 弹性模量随 $\mathrm{pH}$ 降 低略有增加，而材料的损耗模量表现出明显的频率依赖 性，在低频区出现弹性模量与损耗模量交叉的情况，标 志着水凝胶体系的三维网络结构发生了可逆解交联的反 应. 而在中性条件下, 由于酰腙键和双硫键均比较稳定, 因此不存在弹性模量和损耗模量的交叉. 加入苯胺后, 酰腙键的平衡受到影响, 在中性条件下表现出了动态可 逆的特性, 而宏观上实现了自愈(图 4). 碱性条件下, 双 硫键表现出主导的动态可逆特性, 使得水凝胶再次表现 出良好的自愈性. 拉伸实验表明, 自愈前后的水凝胶其 拉伸曲线基本重合, 力学性能不存在明显差异. 由于酰 腙键和双硫键分别具有的酸碱响应和氧化还原响应，使 
得水凝胶在加入酸、碱、氧化还原物质时表现出了溶胶凝胶的转变, 体现了材料的自适性. 这种结合了两个动 态可逆键构筑的自愈性多响应水凝胶代表了自愈性水凝 胶向更丰富的多种类型交联位点的发展.

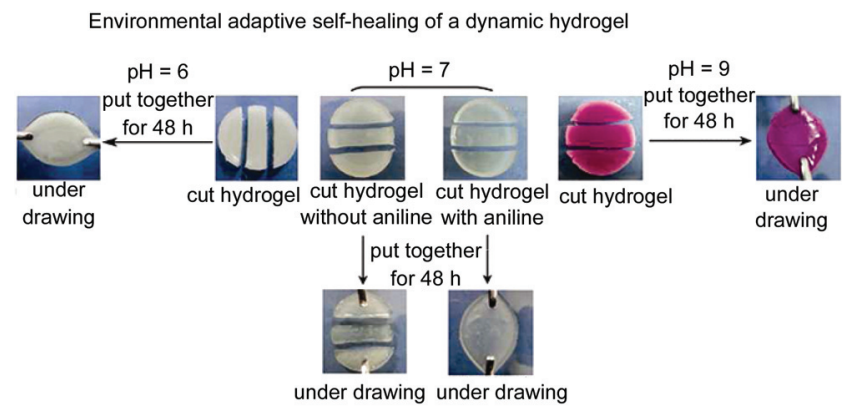

图 4 酰腙键水凝胶在不同环境下的自愈性 ${ }^{[28]}$

Figure 4 Environmental adaptive self-healing properties of a dynamic hydrogel ${ }^{[28]}$

\section{5 亚胺键}

亚胺键作为动态化学键由 Lehn 等做了深入而系统 的研究, 在动态化学中扮演了重要角色. 亚胺键对于不 同反应底物其平衡常数范围很广, 如不同的氨基和醛基 在不同环境下, 包括在不同 $\mathrm{pH}$ 值、不同溶剂体系等条件 下存在丰富的变化情况, 为材料提供了更多的可调控性. 目前广泛应用于生物医用材料中的高分子如壳聚糖、聚 赖氨酸、聚乙烯亚胺、葡聚糖、蛋白质及多肽等物质含 有丰富的氨基, 且在现有的生物相容性高分子, 如聚乙 二醇上通过化学改性修饰上氨基与醛基也相对简单.

Zhang 等 ${ }^{[29]}$ 利用天然高分子壳聚糖上丰富的氨基与 两端被苯甲醛基修饰的聚乙二醇, 在室温下快速形成具 有一定力学强度的动态自愈性水凝胶. 该水凝胶的网络 交联点为亚胺键, 由于水凝胶中大量存在的水分和壳聚 糖溶解时所带的少量酸, 使得体系当中亚胺键的平衡常 数较低 ${ }^{[30,31]}$, 因此存在大量的分子间和分子内的相互交 换而使得整个水凝胶具有动态网络结构 ${ }^{[32]}$, 可以在不 借助外力条件下实现快速愈合. 在水凝胶被打孔后, 界 面提供的微小能量即使水凝胶产生流动相，一段时间后 孔洞消失, 界面变得模糊, 材料完成自愈合(图 5).

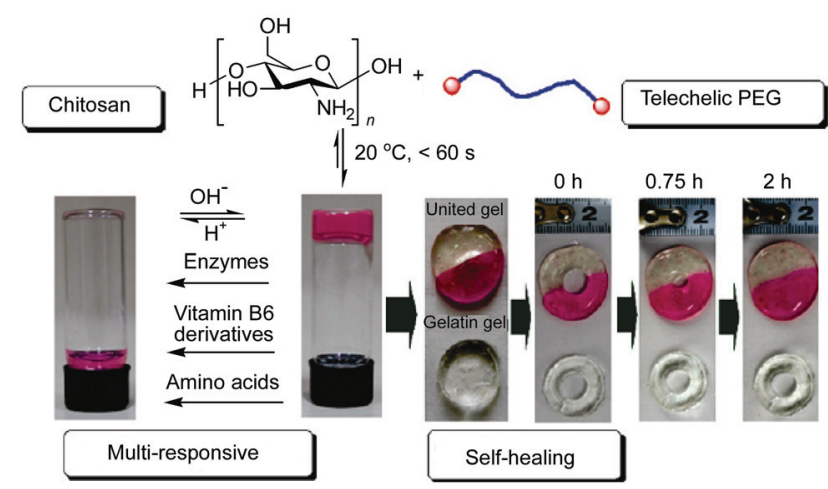

图 5 壳聚糖动态水凝胶的自愈性和多响应性 ${ }^{[29]}$

Figure 5 Multi-responsive and self-healing properties of the dynamic chitosan hydrogel ${ }^{[29]}$
此外，亚胺键的动态特性使得水凝胶在宏观上对外 界环境具有多重响应的特征, 并可在外力方向上产生自 适性的调节. 如体系中加入维生素 B6 衍生物如盐酸吡 哆醛，由于吡哆醛与壳聚糖上的氨基具有更强的结合能 力, 因此原有的交联点被新的动态平衡取代, 使得水凝 胶崩解变成溶胶, 类似的溶胶-凝胶变化也可以通过加 入其他生物活性物质如赖氨酸等富含氨基的小分子调 控动态平衡实现. 此外, 可以加入木瓜蛋白酶等对壳聚 糖骨架进行降解，造成水凝胶的解体. 不同的刺激响应 调控溶胶一凝胶变化，其速率是不同的，使得该水凝胶 具有可控缓释的特性, 并被用于药物模型的控制释放研 究. Zhang 等初步研究了小分子药物模型罗丹明 $\mathrm{B}$ 和大 分子蛋白溶菌酶在不同条件下的释放曲线, 结果表明在 不同的外界刺激条件下，药物模型分子表现出不同的释 放行为, 具有可控释放的特点. 值得一提的是, 大分子 蛋白药物, 即溶菌酶在释放后活性几乎不受影响, 为这 一水凝胶在后续可能的在生物医用材料方面的应用提 供了良好的基础.

对于开发生物医用的水凝胶，良好的生物相容性是 不可或缺的, 细胞实验表明, 该材料生物相容性极佳. 在 后续的试验中, Yang 等 ${ }^{[33]}$ 实现了在这一水凝胶中细胞的 三维培养. 动态水凝胶作为进行细胞三维培养的材料有 着独特的优势 ${ }^{[34,35]}$. 目前大部分的细胞培养都是在二维 水平上进行的. 而在生物组织之中, 细胞是以三维形式 分布的, 因此, 三维细胞培养也是目前细胞培养的新趋 势. 为了更好地模拟细胞生存环境, 就要求在体外培养 细胞时也能具有类似于体内的三维环境. 生物相容性极 好的水凝胶就是一种非常具有应用前景的细胞三维培养 材料. 同时, 动态化学键的存在使得水凝胶内的细胞本 身处于一种动态的环境之中, 体内三维环境的变化会导 致细胞生长状况及形态学的改变, 而动态环境有助于细 胞相互间的接触传递信息以及与环境的互动等. 在动态 水凝胶中, 相对于二维培养环境可以更好地模拟这种变 化, 在更接近生理环境的状态下培养并观察细胞(图 6).

同时，一种新兴的治疗手段——细胞治疗也受到了 人们越来越多的关注. 该疗法将具有特定功能的细胞在 体外进行培养增殖后回输入注入病患体内进行治疗. 而 常规的输入手段是直接将细胞悬液注入静脉, 这势必会 导致注射细胞随血液循环、体液流动而大量流失，极大 地影响了治疗效果. 将细胞包覆于水凝胶内直接对患处 进行注射则可以较好地解决上述的问题，实现更有效的 主动靶向给药. 这时, 自愈性水凝胶的优势得到明显的 体现，如制备过程简易温和，不会造成细胞活性的损失; 注射后破坏的水凝胶在患处实现自愈后保持了材料的 相对完整性，可将细胞固定于患处，并保护细胞免受代 谢系统的破坏.

由于该水凝胶制备简单，同时对多种物质的加入具 有良好的相容性, Zhang 等 ${ }^{[36]}$ 又将功能材料, 如磁性功 能材料纳米四氧化三铁加入该水凝胶体系，制备了可自 

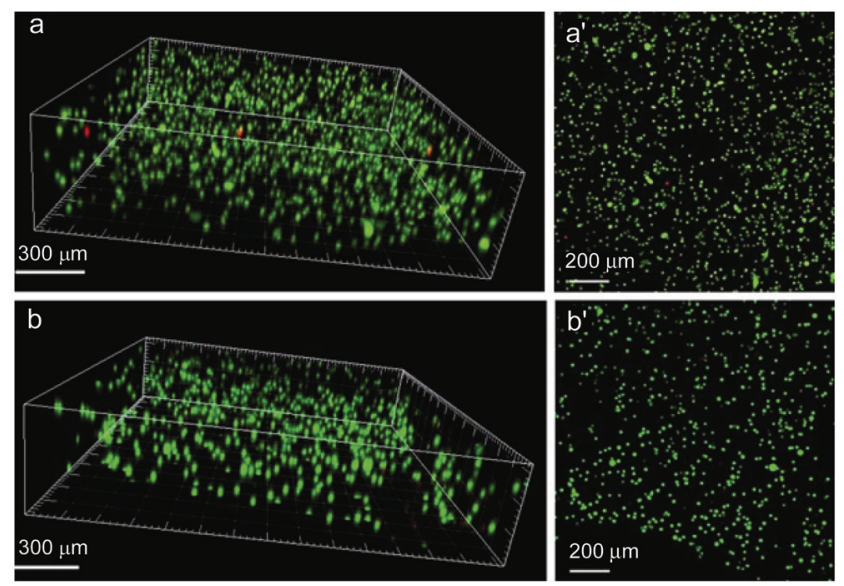

图 6 苂光显微镜下三维视图 (a 和 $b$ ) 和 $Z$ 轴最大投影图 $\left(a^{\prime} \text { 和 } b^{\prime}\right)^{[33]}$

Figure $63 \mathrm{D}$ ( $a$ and $b$ ) and $z$-axis maximum projection ( $a^{\prime}$ and $b^{\prime}$ ) views of confocal microscopy images ${ }^{[33]}$

Cell viability (viable cells: green, dead cells: red) and spatial distribution of HeLa cells encapsulated in the self-healing hydrogels. (a, a') $24 \mathrm{~h}$ culture after gelation. (b, b') $72 \mathrm{~h}$ culture after gelation

愈可变形的磁性水凝胶. 该水凝胶具有类似章鱼的特 性，可通过变形或自身分裂通过比自身狭窄的孔道，并 在之后借由自愈性恢复材料的完整性(图 7). 已证实可 以通过极为简单的方法, 方便地为该水凝胶体系附加其 他的功能, 获得新型功能化水凝胶, 以满足不同的应用 需求, 开发出丰富的生物医用材料.
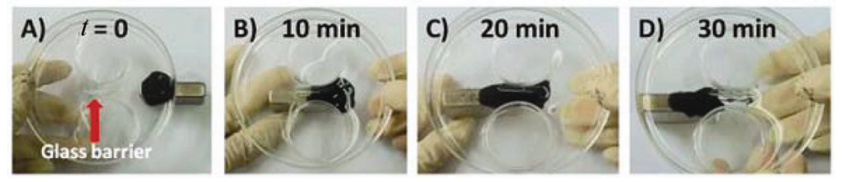

图 7 磁性水凝胶在 N45 磁铁引导下变形通过狭窄通道 ${ }^{[36]}$

Figure 7 Hydrogel passes through a channel with an obstacle in themiddle by shape transformation under the assistance of a N45 magnet $^{[36]}$

$\mathrm{Fu}$ 等 ${ }^{\left[{ }^{[7]}\right.}$ 将二氧化硅溶胶均匀分散在快速形成的水 凝胶中, 水凝胶在这里充当后续的二氧化硅缩聚的骨 架. 冻干后, 得到了具有多级孔结构的壳聚糖 - 聚乙二 醇-二氧化硅 $(\mathrm{CPS})$ 生物复合材料. 细胞毒性评估结果表 明这种生物复合材料几乎是无毒的. 细胞吸附实验 (大 鼠血细胞模型)表明, 这种多级孔结构的 CPS 生物复合 材料与其他材料相比具有更好的细胞吸附能力 (图 8), 吸附在 CPS 生物复合材料表面的细胞几乎保持了完整 形态. 鉴于 CPS 生物复合材料组分(壳聚糖、聚乙二醇、 二氧化硅)良好的生物相容性和材料价廉制备简单的特 性, 这种多级孔结构的有机一无机复合材料具有潜在的 生物学与医疗领域的应用价值, 尤其是作为血吸附与止 血材料等.

基于壳聚糖构建的亚胺键动态可自愈的水凝胶, 其 基体材料为天然高分子壳聚糖, 具有来源广泛、价格低 廉、生物相容性好、抗菌、止血等许多天然优势, 在前 人的研究中有丰富的积累. 而该材料使用的大分子聚乙
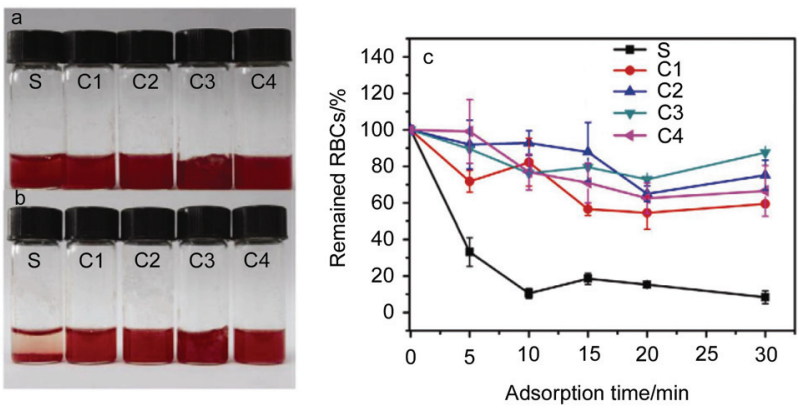

图 8 复合材料血细胞吸附实验在不同时间段(a: $5 \mathrm{~min}, \mathrm{~b}: 30 \mathrm{~min}$ )的 照片对比及血细胞吸附实验曲线(c) $(n=3, P<0.05)$

Figure 8 Optical photograph of cell adsorption by materials after different time (a: $5 \mathrm{~min}, \mathrm{~b}: 30 \mathrm{~min}$ ) and quantitative cell adsorption kinetics results (c) $(n=3, P<0.05)^{[37]}$

S: CPS biohybrid, C1: chitosan-silica hybrid, C2: chitosan-PEG xerogel, C3: chitosan monoliths, and $\mathrm{C} 4$ : porous silica

二醇修饰物作为网络结构交联剂也被证明具有良好的 生物相容性. 同时, 亚胺键的动态化学平衡使得材料具 有多重响应的特性，可应用于开发多功能智能水凝胶材 料. 整个材料制备简单，具有广阔的应用前景.

对于由较强作用如稳定共价键交联形成的材料, “自愈性” 通常则需要外部提供刺激因子，如化学物质、 光、热等能量才能够实现. 例如, 基于可逆二硫键的多 臂高分子 ${ }^{[38]}$ 、光引发修复的三硫代碳酸酯交联网络结 构 ${ }^{[39]}$ 、紫外光修复的壳聚糖-聚氨酯复合材料 ${ }^{[40,41]}$ 、热 引发修复的 Diels-Alder 化学交联结构及基于自由基交 换引发愈合的凝胶材料 ${ }^{[42,43]}$ 等, 在此基于动态非共价的 自愈水凝胶中则不做更多论述.

\section{3 结论与展望}

自愈性水凝胶材料的研究目前是一个热门且令人 兴奋的领域. 大多数水凝胶在受损后不具备自愈合的能 力, 主要是由于其构成骨架刚性过强且不具有动态化学 键. 这种不可自愈性限制了其在多方面的应用, 尤其是 在生物医学方面的应用. 而自愈性水凝胶的引入则有可 能带来生物医用材料领域的一次重大突破.

在各种自愈机理中, 动态化学键已成为人们研究较 多的一个方向. 人们研究了各式各样的自愈水凝胶体系, 如多重氢键作用、金属配位相互作用、疏水相互作用、 酰腙键、亚胺键等都常被应用于其中. 而应用了动态化学 体系的水凝胶材料, 具备了极佳的可调控性能, 对环境 有自适性的响应和调节, 具有潜在的生物应用价值, 如 用于软骨组织工程和可注射性药物载体等. 在可注射性 水凝胶药物载体的研究中, 大多是将前体物质以液体的 形式注入, 再通过改变外界条件, 如温度、 $\mathrm{pH} 、 \mathrm{UV}$ 照射 等使其原位成胶 ${ }^{[44 ~ 47]}$. 虽然一部分文献中的条件变化是 温和的 ${ }^{[48,49]}$ ，但更多情况下，该成胶过程需要依赖外界 条件的急剧变化甚至是引入一些有毒的有机试剂, 或多 或少会对包覆于其中的药物分子或者细胞造成影响与损 
伤. 同时, 体内的成胶过程难以控制, 成胶速度过慢会导 致药物与细胞的逸散损失, 而成胶速度过快也可能会导 致非预期的过早聚合及药物递送的失败. 而应用动态化 学构筑的水凝胶, 可以在体外成胶, 并具有自愈性, 将其 作为可注射水凝胶材料则可以解决上述的成胶动力学等 问题, 有望成为注射性水凝胶材料的发展趋势.

同时, 具有多种相互协调功能的自愈性水凝胶也是 研究趋势. 如本文提及的磁性自愈性水凝胶, 磁性和自 愈性的相互配合使得材料能够在外力驱动下进行远距 离迁移, 这对于生物体内的医用材料极为重要, 而自适 性使得材料能够在外力方向上改变自身形态, 最终穿过 障碍, 这对于生物体内的主动靶向有重要意义. 又如 $\mathrm{Bao}$ 等 ${ }^{[10]}$ 制备了具有压力和弯曲双重感应并具有自愈 性的人工电子皮肤, 他们在高分子网络结构中加入表面 具有特殊纳米结构的镍颗粒赋予材料压电响应, 材料受 损时即可利用多重氢键相互作用在室温下实现快速自 愈, 并且自愈后的材料其导电能力能得到恢复, 即自愈 后的材料除了力学性能其他功能也得以恢复. 这种多功 能的自愈合材料是自愈合领域的新方向, 体现了自愈不 再仅仅局限于物理伤口或力学性能的愈合, 而是一种多 功能相互作用的平衡.

尽管人们对于自愈性水凝胶材料已经做了许多研 究, 但这种水凝胶材料仍然存在着许多亟待解决的问题 和广阔的发展空间. 例如, 虽然自愈性水凝胶材料及其 衍生物具有潜在的生物医用价值, 但目前的研究基本上 还仅限于体外实验, 难以做到体内实验. 而设计生物医 用材料时, 要更多地考虑人体内环境对于材料的要求, 更多时候是希望材料能够自适性地进行智能调节。因 而, 通过进一步地改善材料设计, 获得更强且可控的机 械性能、更好的稳定性以及生物相容性、在体内更好的 动态自愈性及可控生物降解条件下的材料, 将是今后生 物医用水凝胶材料的研究重点.

此外，自愈性水凝胶等自修复材料的研究还处于初 步阶段, 大部分研究重点集中在探索新的材料和体系上, 对于自愈合、自修复等概念的定义比较模糊, 对材料自修 复性能的评价体系尚未建立完善, 尤其是定量的表征和 描述, 大部分还停留在定性的观察和描述上. 因此, 对于 自修复材料的评价体系亟待研究者来共同建立.

\section{作者简介}

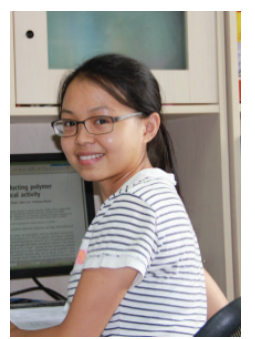

张亚玲, 清华大学化学系 2010 级在读博士生. 研究方向 为功能高分子的合成与应用.

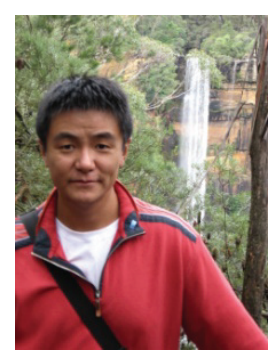

陶磊, 清华大学化学系副研究员. 研究方向为新聚合方 法及功能高分子的合成与应用.

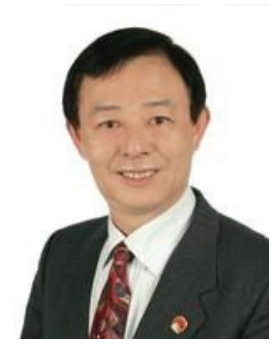

危岩, 清华大学化学系国家特聘“千人计划”讲座教授, 研究兴趣包括高分子合成、表征及性质, 电活性和导电性高分 子, 有机无机杂化/纳米复合材料和溶胶凝胶化学, 酶和细胞 在纳米材料中的固定化, 可控药物释放, 树枝状和手性化合 物的自组装及超分子化学性质, 蛋白质间相互作用, 蛋白质 折叠, 生物传感器等.

\section{References}

[1] Wool, R. P. Soft Matter 2008, 4, 400

[2] Syrett, J. A.; Becer, C. R.; Haddleton, D. M. Polym. Chem. 2010, 1, 978.

[3] Dong, K.; Wei, Z.; Yang, Z.; Chen, Y. Sci. Sin. Chim. 2012, 42, 741 (董坤, 魏到, 杨志禁, 陈咏梅, 中国科学: 化学, 2012, 42, 741.)

[4] Yan, X.; Wang, F.; Zheng, B.; Huang, F. Chem. Soc. Rev. 2012, 41 1621.

[5] Wojtecki, R. J.; Meador, M. A.; Rowan, S. J. Nat. Mater. 2010, 10 , 14.

[6] Chen, Y.; Kushner, A. M.; Williams, G. A.; Guan, Z. Nat. Chem. 2012, 4, 467.

[7] Liu, F.; Urban, M. W. Prog. Polym. Sci. 2010, 35, 3.

[8] Yu, L.; Ding, J. Chem. Soc. Rev. 2008, 37, 1473.

[9] Lehn, J.-M. Chem. Soc. Rev. 2007, 36, 151.

[10] Tee, B. C. K.; Wang, C.; Allen, R.; Bao, Z. Nat. Nanotechnol. 2012 7,825 .

[11] Zhang, H.; Xia, H.; Zhao, Y. ACS Macro Lett. 2012, 1, 1233.

[12] Cui, J.; del Campo Becares, A. Chem. Commun. 2012, 48, 9302.

[13] Haraguchi, K.; Uyama, K.; Tanimoto, H. Macromol. Rapid Commun. 2011, 32, 1253.

[14] Phadke, A.; Zhang, C.; Arman, B.; Hsu, C. C.; Mashelkar, R. A.; Lele, A. K.; Tauber, M. J.; Arya, G.; Varghese, S. Proc. Natl. Acad. Sci. U.S. A. 2012, 109, 4383.

[15] Weng, W. J. Mater. Chem. 2012, 22, 11515.

[16] Burnworth, M.; Tang, L.; Kumpfer, J. R.; Duncan, A. J.; Beyer, F. L.; Fiore, G. L.; Rowan, S. J.; Weder, C. Nature 2011, 472, 334.

[17] Zheng, B.; Wang, F.; Dong, S.; Huang, F. Chem. Soc. Rev. 2012, 41, 1621

[18] Ceylan, H.; Urel, M.; Erkal, T. S.; Tekinay, A. B.; Dana, A.; Guler, M. O. Adv. Funct. Mater. 2012, doi:10.1002/adfm.201202291.

[19] Holten-Andersen, N.; Harrington, M. J.; Birkedal, H.; Lee, B. P. Messersmith, P. B.; Lee, K. Y. C.; Waite, J. H. Proc. Natl. Acad. Sci. U. S. A. 2011, 108, 2651.

[20] Tuncaboylu, D. C.; Sari, M.; Oppermann, W.; Okay, O. Macromolecules 2011, 44, 4997.

[21] Tuncaboylu, D. C.; Sahin, M.; Argun, A.; Oppermann, W.; Okay, O. 
Macromolecules 2012, 45, 1991.

[22] Nakahata, M.; Takashima, Y.; Yamaguchi, H.; Harada, A. Nat. Commun. 2011, 2, 511.

[23] Zhang, M.; Xu, D.; Yan, X.; Chen, J.; Dong, S.; Zheng, B.; Huang, F. Angew. Chem. 2012, 124, 7117.

[24] Appel, E. A.; Loh, X. J.; Jones, S. T.; Biedermann, F.; Dreiss, C. A.; Scherman, O. A. J. Am. Chem. Soc. 2012, 134, 11767.

[25] Fox, J.; Wie, J. J.; Greenland, B. W.; Burattini, S.; Hayes, W.; Colquhoun, H. M.; Mackay, M. E.; Rowan, S. J. J. Am. Chem. Soc. 2012, 134, 5362 .

[26] Xu, Z.; Peng, J.; Yan, N.; Yu, H.; Zhang, S.; Liu, K.; Fang, Y. Soft Matter 2013, 9, 1091.

[27] Deng, G.; Tang, C.; Li, F.; Jiang, H.; Chen, Y. Macromolecules 2010, 43, 1191.

[28] Deng, G.; Li, F.; Yu, H.; Liu, F.; Liu, C.; Sun, W.; Jiang, H.; Chen, Y. ACS Macro Lett. 2012, 1, 275.

[29] Zhang, Y.; Tao, L.; Li, S.; Wei, Y. Biomacromolecules 2011, 12, 2894.

[30] Marin, L.; Simionescu, B.; Barboiu, M. Chem. Commun. 2012, 48, 8778.

[31] Godoy-Alcántar, C.; Yatsimirsky, A. K.; Lehn, J. M. J. Phys. Org. Chem. 2005, 18, 979.

[32] Kovarícek, P.; Lehn, J. M. J. Am. Chem. Soc. 2012, 134, 9446.

[33] Yang, B.; Zhang, Y.; Zhang, X.; Tao, L.; Li, S.; Wei, Y. Polym. Chem. 2012, 3, 3235.

[34] Gillette, B. M.; Jensen, J. A.; Wang, M.; Tchao, J.; Sia, S. K. Adv. Mater. 2010, 22, 686.

[35] Lim, H. L.; Chuang, J. C.; Tran, T.; Aung, A.; Arya, G.; Varghese,
S. Adv. Funct. Mater. 2011, 21, 55.

[36] Zhang, Y.; Yang, B.; Zhang, X.; Xu, L.; Tao, L.; Li, S.; Wei, Y. Chem. Commun. 2012, 48, 9305.

[37] Fu, C.; Wang, S.; Feng, L.; Liu, X.; Ji, Y.; Tao, L.; Li, S.; Wei, Y. Adv. Healthcare Mater. 2012, 2, 302.

[38] Yoon, J. A.; Kamada, J.; Koynov, K.; Mohin, J.; Nicolaÿ, R.; Zhang, Y.; Balazs, A. C.; Kowalewski, T.; Matyjaszewski, K. Macromolecules 2011, 45, 142.

[39] Amamoto, Y.; Kamada, J.; Otsuka, H.; Takahara, A.; Matyjaszewski, K. Angew. Chem. 2011, 123, 1698.

[40] Ghosh, B.; Chellappan, K. V.; Urban, M. W. J. Mater. Chem. 2011, $21,14473$.

[41] Ghosh, B.; Urban, M. W. Science 2009, 323, 1458

[42] Imato, K.; Nishihara, M.; Kanehara, T.; Amamoto, Y.; Takahara, A.; Otsuka, H. Angew. Chem., Int. Ed. 2012, 51, 1138

[43] Yuan, C.; Rong, M. Z.; Zhang, M. Q.; Zhang, Z. P.; Yuan, Y. C. Chem. Mater. 2011, 23, 5076.

[44] Jeong, B.; Bae, Y. H.; Lee, D. S.; Kim, S. W. Nature 1997, 388, 860.

[45] Mano, J. F. Adv. Eng. Mater. 2008, 10, 515.

[46] Petka, W. A.; Harden, J. L.; McGrath, K. P.; Wirtz, D.; Tirrell, D. A. Science 1998, 281, 389.

[47] Chung, H. J.; Park, T. G. Nano Today 2009, 4, 429.

[48] Chang, G.; Ci, T.; Yu, L.; Ding, J. D. J. Controlled Release 2011, $156,21$.

[49] Yu, L.; Chang, G. T.; Zhang, H.; Ding, J. D. Int. J. Pharm. 2008, 348,95 . 